\title{
Use and accuracy of magnetic resonance imaging (MRI) staging of network treated endometrial cancer (EC): an audit and comparison to best evidence
}

\author{
J Anderson $^{1}$, S Shanbhagㅁ, D Cruikshank ${ }^{2}$ \\ Sri Lanka Journal of Obstetrics and Gynaecology 2009; 31: 104-107
}

\begin{abstract}
Introduction: As a result of national and international guidelines, in recent years treatment of endometrial carcinoma (EC) has become more centralised. In the UK this has led to the development of regional cancer networks, with sub-specialists gynaecological-oncologists leading treatment in regional centres. A tool in appropriate triage of patients to treatment at central or peripheral units is magnetic resonance imaging (MRI).
\end{abstract}

Objective: To assess if MRI is an effective tool in triaging place of treatment within the Cancer Network.

Methods: Between March 2006 and December 2007 an audit was undertaken, comparing MRI and histology reports in women with tissue diagnosis of EC. The MRI reports were matched to histology and International Federation of Gynecology and Obstetrics (FIGO) staging was compared. A review of guidance and expected accuracy was also performed.

Results: Forty-three cases were analysed. Sensitivity or MRI for overall myometrial invasion was $88 \%$, a positive predictive value (ppv) of $94 \%$ and a negative predictive value (npv) of $56 \% .65 .2 \%$ of reports commented on cervical involvement clearly, which was accurate in $44.2 \%$ cases. For cervical involvement, sensitivity was $57 \%$, specificity $75 \%$, ppv $44 \%$ and npv $83 \%$.

Conclusions: Practical reporting in our Network structure falls short of expectations. Standardisation of reporting under named lead

\footnotetext{
1 Senior Registrar,

2 Consultant, Department of Gynaecological Oncology, The James Cook University Hospital, Lead Centre for North of England (South) Cancer Network, Middlesbrough, UK.
}

Corresponding author: J Anderson

E-mail: johnanderson3@nhs.net radiologists may help improve accuracy and thus the utility of MRI to the regional cancer network.

Key words: MRI staging, endometrial cancer.

\section{Introduction}

Traditionally patients in the UK diagnosed with endometrial cancer (EC) were operated at the hospital that they initially presented. In 1995, the Calman-Hine report, a publication of the Department of Health, UK, led to sub-specialisation of gynaecological cancer surgery. Increasingly general gynaecologists have done less cancer surgery and expert recommendation has advised concentration of oncological surgery expertise in particular units ${ }^{1}$. This centralisation has led to the development of regional cancer networks within the UK. Each network consists of peripheral units serving a local population, with a specialised cancer centre providing tertiary services for specialist oncology treatment, to which so-called "peripheral units" refer.

Accordingly, in EC all G3 as well as those tumours deemed to be Stage $1 \mathrm{C}$ and above are meant to be treated at the centre, while lower stage/risk cancers being treated more locally in the diagnosing unit. IOG (Improving Outcome Guidelines) recommends pre-operative imaging to stage the EC and thus determine the place of treatment for these patients within cancer networks ${ }^{2}$.

The use of ultrasound as a tool to assess myometrial invasion has been advocated by many for this purpose ${ }^{3}$. In the last few years magnetic resonance imaging (MRI) has increasingly been used for staging endometrial cancer following Royal College of Radiologists recommendations ${ }^{4}$. FIGO staging of endometrial cancer is based on assessment of myometrial, cervical and parametrial invasion but in addition MRI allows an assessment of lymph node status $^{3}$. Accuracy of MRI staging has been reported in the literature as between $52-97 \%{ }^{5}$. American College of Radiology (ACR) appro-priateness criteria ${ }^{6}$ rate MRI as superior in the diagnostic work-up of newly diagnosed EC compared to CT and ultrasound (US) for assessing myometrial invasion, endocervical 
involvement and overall staging. MRI and CT were both equally superior to US for lymph node assessment.

Since 2007 it has been standard practice to use MRI for cases of endometrial carcinoma in the North of England (South) Cancer Network, which has a catchment area of 1.5 million and four peripheral hospitals referring patients to one central unit at the James Cook University Hospital (JCUH), Middlesbrough (Table 1). MRI is used to determine the place of treatment using pre-operative imaging findings ${ }^{7}$. These images are taken pre operatively after histological diagnosis from an endometrial biopsy and assessed using the criteria stated earlier and patients triaged accordingly. The role therefore of the smaller units is to diagnose and assess patients, treat those with less severe disease (less than FIGO 1B) and refer those with more severe disease (Table 2).

\section{Objectives}

1. To determine if MRI reporting is complete for confirmed cases of endometrial cancer within a Cancer Network.

2. To correlate MRI and final histological FIGO staging.

\section{Audit Standards}

Our audit standards were based upon the information required to accurately stage the cancer using the FIGO staging system. These were;

1. Every MRI report should clarify from its content possible FIGO stage from data on myometrial invasion, cervical involvement as well as lymph node status.

2. MRI should accurately allow patients to be appropriately triaged for treatment as per IOG guidance.

\section{Materials and methods}

Between March 2006 and December 2007 we identified a cohort of women diagnosed with endometrial cancer, who were investigated with MRI. Data were collected from MRI and histology reports. The MRI reports were matched to histology reports and the data was compiled into a Microsoft Excel@ spreadsheet. Data collected included case demographics, clinical diagnosis, referring hospital, MRI report, treatment location, and histology report. Analysis was undertaken using Microsoft Excel@ and SPSS. If FIGO stage was not explicitly stated, but the report contained adequate detail, it was interpreted at consultant level.

\section{Results}

Forty-seven women were identified, 4 were excluded, 2 of which had cervical cancers and the other two 2 because MRI could not be reported (claustrophobia, excessive artefact). Of the 43 cases analysed, mean age was 63 years and only eleven MRI reports explicitly gave a FIGO stage and 29 implied it. Table 1 gives a breakdown of reports by referring unit in the network.

\section{Table 1. Cases and FIGO staging in MRI} report by referring unit

\begin{tabular}{lccc}
\hline $\begin{array}{l}\text { Referring and } \\
\text { MRI Unit }\end{array}$ & Cases (\%) & $\begin{array}{c}\text { FIGO } \\
\text { Explicit }\end{array}$ & $\begin{array}{c}\text { FIGO } \\
\text { implied }\end{array}$ \\
\hline $\begin{array}{l}\text { Darlington } \\
\text { JCUH (and }\end{array}$ & $10(23 \%)$ & 0 & 10 \\
$\begin{array}{l}\text { Northallerton) } \\
\text { North Tees }\end{array}$ & $14(33 \%)$ & 2 & 10 \\
Scarborough & $2(5 \%)$ & 7 & 9 \\
Total & 43 & $11(25 \%)$ & $29(67 \%)$
\end{tabular}

The International Federation of Obstetrics and Gynaecology (FIGO, 2002.) gives the following staging for endometrial carcinoma.

Stage I endometrial cancer is carcinoma confined to the corpus uteri

1. IA confined to endometrium

2. IB invasion to less than half of myometrium

3. IC invasion beyond half of myometrium

Stage II involves the corpus and the cervix, but has not extended outside the uterus

1. Stage IIA is endocervical glandular involvement only

2. Stage IIB is cervical stromal invasion

Stage III extends outside of the uterus but is confined to the true pelvis

1. Stage IIIA is invasion of serosa or adnexa or positive peritoneal cytology and possibly more than one of these

2. Stage IIIB is vaginal metastases

3. Stage IIIC is metastases to pelvic or para-aortic lymph nodes or both 
Stage IV is involvement of the bladder or bowel mucosa or distant metastasis

1. Stage IVA is involvement of bowel or bladder mucosa

2. Stage IVB is distant metastases including nodes in the abdomen or inguinal region

A further grouping with prognostic significance is possible with FIGO approval, based on degree of tumour differentiation as follows:

1. G1 is $5 \%$ or less of a nonsquamous or nonmorular solid growth pattern

2. G2 is 6 to $50 \%$ of a nonsquamous or nonmorular solid growth pattern
3. G3 is over 50 and of a nonsquamous or nonmorular solid growth pattern.

Eighty one percent commented on myometrial invasion clearly, which was accurate in $53.5 \%$ cases. Sensitivity for overall myometrial invasion was $88 \%$, specificity $71 \%$, a positive predictive value (ppv) of $94 \%$ and a negative predictive value (npv) of $56 \%$. Sensitivity for depth of invasion was $71 \%$. $65.2 \%$ commented on cervical involvement clearly, which was accurate in $44.2 \%$ cases. For cervical involvement, sensitivity was $57 \%$, specificity $75 \%$, ppv $44 \%$ and npv $83 \%$. Ninety five percent commented on lymph node status clearly.

Table 3 compares MRI with histological findings

Table 3. Correlation of MRI and histological FIGO staging

\begin{tabular}{|c|c|c|c|c|c|c|c|c|}
\hline MRI & & & & & & & & \\
\hline Histol FIGO & $1 \mathrm{a}$ & $1 b$ & 1c & $2 a$ & $2 b$ & $3 a$ & $3 c$ & Total \\
\hline FIGO 1a & 4 & & & 1 & & & & 5 \\
\hline FIGO $1 b$ & 3 & 1 & 6 & & 1 & & & 12 \\
\hline FIGO 1c & 2 & 1 & 5 & & & 3 & & 11 \\
\hline FIGO 2a & & & 1 & & & & & 1 \\
\hline FIGO $2 b$ & 2 & & 2 & & 1 & 1 & 1 & 9 \\
\hline FIGO 3c & & & 1 & & & & 1 & 2 \\
\hline FIGO $4 b$ & & & & & 2 & 1 & & 3 \\
\hline Total & 11 & 2 & 15 & 1 & 4 & 5 & 2 & 43 \\
\hline
\end{tabular}

MRI accurately staged 30\%, overstaged 33\% and understaged 38\% of cases. In 3 cases, the reporting was insufficient to stage. The sensitivity and positive predictive value (ppv) for MRI to accurately stage endometrial cancers was 0.46 and 0.44 respectively.

Table 4 shows the outcome of triaging based upon MRI reporting.

Table 4. Correlation of MRI and histological FIGO staging - appropriate triage

\begin{tabular}{|c|c|c|c|c|c|c|c|c|}
\hline \multicolumn{9}{|c|}{ MRI FIGO } \\
\hline Histol FIGO & $1 \mathrm{a}$ & $1 b$ & 1c & $2 a$ & $2 b$ & $3 a$ & $3 c$ & Total \\
\hline FIGO 1a & 4 & & & 1 & & & & 5 \\
\hline FIGO 1b & 3 & 1 & 6 & & 1 & & & 11 \\
\hline FIGO 1c & 2 & 1 & 5 & & & 3 & & 11 \\
\hline FIGO 2a & & & 1 & & & & & 1 \\
\hline FIGO 2b & 2 & & 2 & & 1 & 1 & 1 & 7 \\
\hline FIGO 3c & & & 1 & & & & 1 & 2 \\
\hline FIGO $4 b$ & & & & & 2 & 1 & & 3 \\
\hline Total & 11 & 2 & 15 & 1 & 4 & 5 & 2 & 40 \\
\hline
\end{tabular}


Only $30 \%$ were accurately staged and appropriately triaged, $13 \%$ were overstaged but managed in the appropriate place, $8 \%$ understaged but managed in the appropriate place, $13 \%$ understaged and inappropriately managed in the cancer unit, $18 \%$ understaged and appropriately managed in the cancer centre and $20 \%$ overstaged and inappropriately managed in the centre.

\section{Discussion}

In order to triage endometrial cancer according to staging as per IOG recommendations, MRI reports should include data on degree of myometrial invasion, cervical involvement and lymph node status. In our audit, reporting was incomplete in $30 \%$ of cases, mainly for cervical involvement reporting. Most reports however commented upon myometrial invasion and lymph node involvement. Strictly speaking, reports lacking any of this explicit information could not be used to stage accurately. It was only with subsequent gynae-oncology consultant review of individual reports that enabled interpretation of a FIGO stage. Overall, only $25 \%$ of reports gave a FIGO stage, $67 \%$ of reports implied it but 3 could not be staged. Thus MRI reports would have generally failed for non gynae-oncology medical staff in making a decision about place of treatment for a patient.

The results also show that MRI failed to accurately predict FIGO stage in more than $50 \%$ of cases. In 2 out of 3 cases, MRI either understaged or overstaged the disease. Only $30 \%$ of women were accurately staged and hence appropriately triaged by the MRI report. However, 39\% women with incorrect MRI reporting were also managed in the appropriate place, because the staging fell within the referring cutoff (e.g. stage $1 \mathrm{~b}$ or less and patient was stage $1 \mathrm{~b}$, or stage $1 c$ and above e.g. patient had a stage $2 c$ tumour). Thirty-two percent were managed in the wrong place, with $13 \%$ operated at the referring unit rather than the centre. Twenty percent were inappropriately managed at the cancer centre, thus potentially increasing their anxiety and burden for their family.

Our results also compare unfavourably to transvaginal ultrasound (TVS) which was used prior to the use of MRI within this Cancer Network in accordance with IOG. An audit in 20068 showed a sensitivity of $63 \%$ for myometrial invasion in women with suspected endometrial cancer. Our results show that MRI accurately predicted myometrial invasion in $58 \%$ women. The disadvantage of TVS is the inability to examine lymph node status, and hence comparison of MRI and TVS for accurate staging is limited.

\section{Conclusion}

Practical reporting in our Network structure falls short of peer group expectations. Structured minimum datasets as recommended by the RCR would standardise MRI reporting for patients with EC. It is our supposition that named lead radiologists should develop an ongoing expertise in MRI imaging for EC patients so that results for this modality can be improved to what is reportedly achievable. Then MRI might become a more useful tool for the effective triage of cancer patients between centre and unit.

\section{References}

1. A policy framework for commissioning cancer services: A report by the Expert Advisory Group on Cancer to the Chief Medical Officers of England and Wales. ("CalmanHine Report"). The Expert Advisory Group on Cancer to the Chief Medical Officers of England and Wales. 1st April 1995. Crown Copyright.

2. National Health Service Executive. Guidance on Commissioning Cancer Services Improving Outcomes in Gynaecological Cancers, Department of Health. 1999.

3. FIGO Staging Classifications and Clinical Practice for Gynaecological Cancers. Benedict JL, Denny L, Jones HW, Kavanagh J, Kitchener H, Kohorn E \& Thomas G. 2006.

4. http://www.rcr.ac.uk/docs/oncology/pdf/ Cross_Sectional_Imaging_12.pdf [Royal College of Radiologists, access valid as of 01/07/2009]

5. Barwick T, Rockall A, Barton D and Sohaib S. Imaging of endometrial adenocarcinoma. Clinical Radiology 2006; 61: 545-55.

6. American college of radiology ACR appropriateness criteria. Endometrial cancer of the uterus. Date of origin 1999, last review date 2005.

7. Cancer Care Alliance of Teesside, South Durham, and North Yorkshire, Gynaecological Oncology Working Group Guidelines for the Management of Gynaecological Malignancies. 2004 Edition.

8. Audit of compliance with guidance: Endometrial cancer and myometrial invasion. Chattopadhayay S, Al-Husban N, Giannopoulos T, Cruikshank D J and Twigg J. James Cook University Hospital, 2006. 